\title{
Embryonic and adult isoforms of XLAP2 form microdomains associated with chromatin and the nuclear envelope
}

\author{
Magdalena Chmielewska • Magda Dubińska-Magiera • Mirosław Sopel • \\ Dorota Rzepecka • Christopher J. Hutchison • Martin W. Goldberg • \\ Ryszard Rzepecki
}

Received: 10 August 2010 / Accepted: 12 January 2011 /Published online: 24 February 2011

(C) The Author(s) 2011. This article is published with open access at Springerlink.com

\begin{abstract}
Laminin-associated polypeptide 2 (LAP2) proteins are alternatively spliced products of a single gene; they belong to the LEM domain family and, in mammals, locate to the nuclear envelope (NE) and nuclear lamina. Isoforms lacking the transmembrane domain also locate to the nucleoplasm. We used new specific antibodies against the N-terminal domain of Xenopus LAP2 to perform immunoprecipitation, identification and localization studies during Xenopus development. By immunoprecipitation and mass spectrometry (LC/MS/MS), we identified the embryonic isoform XLAP2 $\gamma$, which was downregulated during development similarly to XLAP2 $\omega$. Embryonic isoforms XLAP2 $\omega$ and XLAP2 $\gamma$ were located in close association with chromatin up to the blastula stage. Later in development, both embryonic isoforms and
\end{abstract}

Electronic supplementary material The online version of this article (doi:10.1007/s00441-011-1129-2) contains supplementary material, which is available to authorized users.

This work was partially supported by the European Social Fund, by Lower Silesia Research Grants for PhD students and by Erasmus training fellowships to M. C. and M. D.-M. at the Integrative Cell Biology Laboratory, University of Durham.

\footnotetext{
M. Chmielewska $\cdot$ M. Dubińska-Magiera $\cdot$ D. Rzepecka $\cdot$

R. Rzepecki $(\bowtie)$

Laboratory of Nuclear Proteins, Faculty of Biotechnology,

University of Wrocław,

Przybyszewskiego 63/77,

51-148 Wrocław, Poland

e-mail: rzepecki@ibmb.uni.wroc.pl

M. Sopel

Department of Pharmaceutical Biology and Botany,

Wrocław Medical University,

Jana Kochanowskiego 10,

51-601 Wrocław, Poland
}

the adult isoform XLAP2 $\beta$ were localized in a similar way at the NE. All isoforms colocalized with lamin B2/B3 during development, whereas XLAP $2 \beta$ was colocalized with lamin B2 and apparently with the F/G repeat nucleoporins throughout the cell cycle in adult tissues and culture cells. XLAP2 $\beta$ was localized in clusters on chromatin, both at the NE and inside the nucleus. Embryonic isoforms were also localized in clusters at the NE of oocytes. Our results suggest that XLAP2 isoforms participate in the maintenance and anchoring of chromatin domains to the NE and in the formation of lamin B microdomains.

Keywords XLAP2 - Nuclear envelope - Nuclear lamina . Chromatin $\cdot$ Cell cycle $\cdot$ Development $\cdot$ Xenopus laevis (Anura)

\section{J. Hutchison - M. W. Goldberg}

Integrative Cell Biology Laboratory, School of Biological and Biomedical Sciences, The University of Durham,

South Road,

Durham DH1 3LE, UK

Present Address:

M. Chmielewska

Department of Histology and Embryology,

Wrocław Medical University,

Chałubinskiego 6A,

50-368 Wrocław, Poland 


\section{Introduction}

Lamin-associated polypeptide 2 (LAP2) proteins belong to the family of LEM domain proteins associated with the inner nuclear envelope (NE) and nuclear lamina (Dorner et al. 2007; Schirmer and Foisner 2007; Wagner and Krohne 2007; Zaremba-Czogalla et al. 2011). They are alternatively spliced products of a single gene and act as integral membrane or nucleoplasmic proteins (Harris et al. 1994). Six LAP2 isoforms have been identified in mammals $(\alpha, \beta$, $\gamma, \delta, \varepsilon, \zeta)$. The LAP2 proteins are widely expressed and evolutionarily conserved in vertebrates with up to $90 \%$ sequence identity in the regions responsible for the function of the protein. The N-terminal part (187 amino acids [aa]), which is common to all LAP2 isoforms, contains the LEM domain (aa 111-152), a structural motif responsible for interaction with BAF (barrier to autointegration factor; (Furukawa 1999; Shumaker et al. 2001), and the LEM-like domain (aa 1-50) interacting directly with chromatin (Cai et al. 2001). The binding sites for lamin B (aa 298-373), the germ-cell-less (GCL) protein (Nili et al. 2001) and HA95 protein (Martins et al. 2003) lie in the variable region of LAP2. Depending on the splicing pattern and presence of the transmembrane domain in the C-terminus, LAP2 proteins are integral membrane $(\beta, \gamma, \delta, \varepsilon)$ or intra-nuclear proteins $(\alpha, \zeta)$ and play diverse roles in the cell nucleus (Shaklai et al. 2008). LAP2 expression differs in different cell types: LAP2 $\beta$ and $\gamma$ are found in somatic cells, whereas LAP $2 \alpha$ is intensively produced in proliferating cells and is the only form present in mature sperm (Alsheimer et al. 1998).

LAP2 proteins play an important role in mammals, in the attachment of chromatin to the NE and nuclear lamina filaments during interphase and in nuclear reassembly after mitosis. LAP2 proteins interact with A and $\mathrm{B}$ type lamins and, through the LEM domain, also with BAF by interconnecting lamin filaments with chromatin inside the nucleus (LAP2 $\alpha$, LAP2 $\zeta$; Shaklai et al. 2008) and at the NE (LAP $2 \beta, \operatorname{LAP} 2 \gamma, \operatorname{LAP} 2 \varepsilon$, LAP2 $\delta$ ). LAP $2 \alpha$-lamin A/C protein complexes are important for the retention of retinoblastoma protein in the cell nucleus (Gant et al. 1999; Markiewicz et al. 2002; Pekovic et al. 2007; Yang et al. 1997).

LAP2 $\beta$ interacts directly with mouse transcriptional repressor protein, GCL (Nili et al. 2001), and together with its binding partners is able to repress the activity of the E2F5-DP3 transcription factor. LAP2 $\beta$ directly interacts, through the same region as GCL, with histone deacetylase 3 (HDAC3) and thus might be involved in the regulation of chromatin organization (Somech et al. 2005). The Nterminal part of human LAP $2 \beta$, comprising the LEM domain and lamin-binding region (aa 1-408) and the common domain only (aa 1-187) are able to block in vitro the formation of pronuclei (Gant et al. 1999).
In Xenopus, five cDNA sequences have been identified coding for LAP2 proteins and have been translated in silico to several isoforms. Two LAP2 isoforms of 557 and 518/ 519 aa contain a transmembrane domain, whereas the isoform of 409 aa lacks this domain (Gant et al. 1999; Lang et al. 1999). Three different polypeptides have also been recognized in immunoblots with anti-human MAN serum and antibodies generated against aa 255-429 of the cDNA clone named XLAP2 $\beta$ (see Fig. 1c for details of the domain/exon structure of cloned XLAP2 cDNAs; note that isolated cDNA clone 3 neither possess a typical lamin binding domain nor a transmembrane domain). The in vitro expression of XLAP $2 \beta$ cDNA results in the synthesis of a 66-kDa polypeptide confirming that this protein is indeed XLAP2 $\beta$ (Lang and Krohne 2003; Lang et al. 1999). The somatic XLAP $2 \beta$ polypeptide is the only one found in adult animals, whereas polypeptides of $86 \mathrm{kDa}$ and $40 \mathrm{kDa}$ are present in oocytes and eggs (Lang and Krohne 2003; Lang et al. 1999) but no data for the expression of the 40$\mathrm{kDa}$ polypeptide during embryogenesis have ever been shown. The N-terminal common fragment of XLAP2, when added to an in vitro nuclear assembly, inhibits chromatin decondensation and nuclear growth similar to the human Nterminal fragment (Gant et al. 1999). A later report has also demonstrated the coprecipitation of XLAP2 $\beta$ protein with lamins B1, B2 and A from A6 Xenopus cells (Lang and Krohne 2003). The common N-terminal domain of XLAP2 (aa 1-165) interacts with BAF and BAF-DNA complexes. Moreover, bacterially expressed Xenopus LAP2 cDNA clones $2(\omega), 4(\beta)$ and 3 bind to BAF with different affinities (Shumaker et al. 2001). Embryonic XLAP2 $\omega$ and/or XLAP2 $\gamma$ proteins interact in vitro with a spindle assembly factor, TPX2 protein, and participate through this protein in the proper assembly of postmitotic nuclei in the Xenopus in vitro nuclear assembly system $\left(\mathrm{O}^{\prime} \mathrm{Brien}\right.$ and Wiese 2006). Little or no data on the subcellular localization and developmental regulation of the expression and distribution of particular XLAP2 isoforms have been reported so far. Moreover, no precise data are available on the identification of particular XLAP2 isoforms and relationships between isolated cDNA clones and identified peptides. In addition, the exact location of XLAP2 proteins in the cell nucleus and at the NE during development and in adult cells is unknown.

In this study, we have taken advantage of newly prepared, affinity-purified antibodies raised against the common N-terminal fragment (1-165 aa) of XLAP2 proteins in our laboratory (Salpingidou et al. 2008). By mass spectrometry, we have identified the presence of two early-development-specific isoforms: XLAP2 $\omega$ (86 kDa) and XLAP2-40 $\mathrm{kDa}$. We have demonstrated that the subcellular localization of embryonic XLAP2 proteins is similar to the location of the adult isoform (XLAP2 $\beta$ ) and 

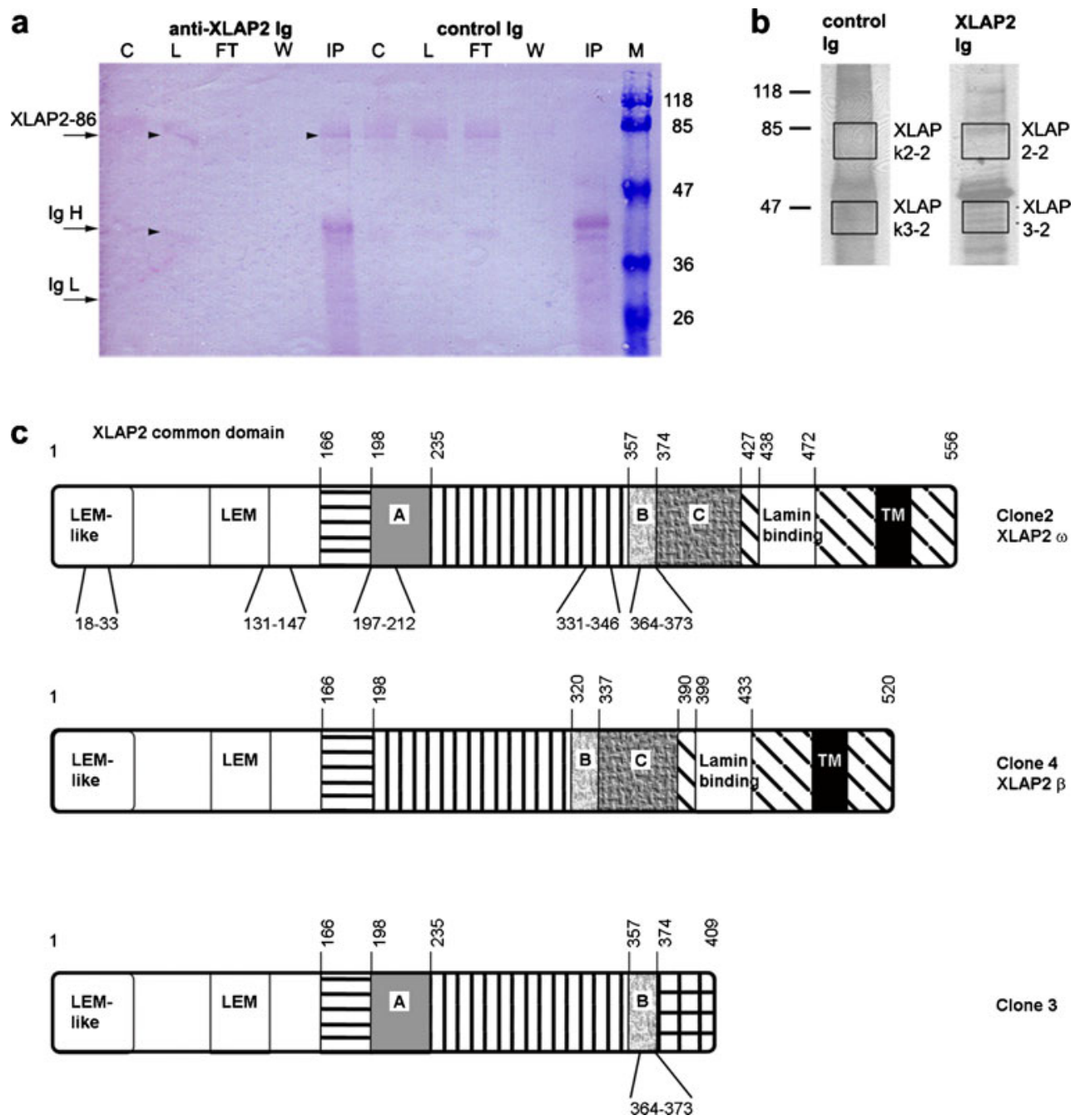

Clone 3

Fig. 1 Identification of XLAP2 $\omega$ and XLAP2 $\gamma$ polypeptides immuno-isolated from Xenopus egg extract. a Immunoblot from the immuno-isolation experiment with control and anti-XLAP2 Igs; staining with XLAP2-specific antibodies. Of the total material, $10 \%$ was loaded onto each lane (arrowheads positions of the $86-\mathrm{kDa}$ XLAP $2 \omega$ and $40-\mathrm{kDa}$ XLAP2 $\gamma$ bands in control egg extract in the $C$ lane and of isolated XLAP2 $\omega$ polypeptide in the IP lane, $C$ control egg extract, $I g-H$ heavy immunoglobulin chain, $I g-L$ light immunoglobulin chain, $L$ loaded egg extract, $W$ wash, $F T$ flow through, $I P$ immunoprecipitated proteins, $M$ molecular-weight markers). b Fragments of silver-stained gel containing protein samples from the pulldown reaction $(90 \%$ of the material was loaded). Gel pieces from boxes were excised and subjected to mass spectrometry analysis (LC/ MS/MS). Molecular masses of reference proteins (in $\mathrm{kDa}$ ) are shown. c Representations of XLAP2 protein isoforms translated in vitro from

that all isoforms associate similarly with anaphase chromatin. We have also demonstrated that XLAP2 $\beta$ is present at the NE and at chromatin inside the nucleus in the form of clusters and microdomains. Similarly, both embryonic isoforms, viz. XLAP2 $\omega$ and XLAP2 $\gamma$, locate at the NE in clusters forming microdomains. All three isoforms colocalize with lamin B2, lamin B3 and, apparently, Nup62 (F/G repeat nucleoporin) in development and throughout the cell cycle.
cDNA sequences found in Xenopus: XLAP2 clone 2 (AN: AF048815); XLAP2 clone 3 (AN: AF048816); XLAP2 clone 4 (AN: AF048817, Gant et al. 1999); XLAP2ß (AN: Y17861, Lang et al. 1999); XLAP2w (AN: AJ514937, Schoft et al. 2003). Identical regions are shaded similarly. Numbers above each representation indicate the amino-acid positions of domains. Sequences marked with letters are putative Xenopus-specific exons (Gant et al. 1999), which are absent from mammals and are positioned between exons 5 and 6 (A) or 8 and 9 of mouse cDNA (Berger et al. 1996). The laminbinding domain is according to (Lang and Krohne 2003). Tryptic peptides identified by using LC/MS/MS are given below each representation. The $86-\mathrm{kDa}$ polypeptide was identified as the translation product of two cDNAs: XLAP2 clone 2 (AF048815) and XLAP2 $\omega$ (AJ514937). The 40-kDa polypeptide was determined as a member of XLAP2 family

\section{Materials and methods}

Plasmids and cDNAs

Plasmid pET23a coding for the $165 \mathrm{~N}$-terminal amino acids of XLAP2 as a His-tag fusion protein was the kind gift of Prof. K. Wilson, Baltimore, USA. Sequence comparison was performed on the following XLAP2 cDNAs: $\beta$ 
(Y17861), clone 2 (AF048815), clone 3 (AF048816), clone 4 (AF048817) and $\omega$ (AJ514937).

Cells, tissues and embryos

Xenopus XTC cells were grown in 54\% L-15 Leibovitz medium containing $10 \%$ fetal bovine serum, $2 \mathrm{mM}$ Lglutamine, $10 \mathrm{I} . \mathrm{U} . / \mathrm{ml}$ penicillin, $10 \mu \mathrm{g} / \mathrm{ml}$ streptomycin, $0.025 \mu \mathrm{g} / \mathrm{ml}$ amphotericin B at $22-26^{\circ} \mathrm{C}$ under normal air conditions. Tissue samples ( $3 \mathrm{~mm}$ in diameter) were dissected out from male frogs and either snap-frozen in liquid nitrogen and stored at $-70^{\circ} \mathrm{C}$ (for biochemical studies) or fixed for microscopy. Tissue extracts were prepared from samples powdered in a RetschMill homogenizer in liquid nitrogen, boiled in extraction buffer and recovered by centrifugation. Embryos were directly homogenized in SDS-sample buffer and boiled. Embryonic stages were classified according to Nieuwkoop and Faber (1994).

Antibodies and antibody purification

The anti-XLAP2 serum was produced in rabbit by immunization with the bacterially expressed, affinitypurified N-terminal (1-165 aa) fragment of the XLAP2 protein. The specific immunoglobulins were purified from full serum on antigen covalently bound to resin (Rzepecki et al. 1998).

The following antibodies were used: rabbit anti-XLAP2 serum (1:250 for immunoblotting [WB], 1:100 for immunofluorescence and immunohistochemistry [IF]), affinitypurified rabbit anti-XLAP2 IgGs (1:100 WB, 1:60 IF), mouse monoclonal antibodies against Xenopus lamins B2/ B3, L6-5D5 (Meier et al. 1991; 1:25 IF), and against B2, L7-8 C6 (Ralle et al. 2004; 1:60 IF; kindly supplied by Prof. Reimer Stick), antibody (Ab) 414 (anti-p62; 1:30 IF; Covance), anti-phosphorylated-histone H3 RR002 (1:400; Upstate Biotechnology), Ac-40 actin Ab (1:800 WB; Sigma, USA), anti- $\beta$-actin ab8224 (1:4000 WB; Abcam) and rat monoclonal anti-alpha-tubulin YL1/2 (1:60 IF; Serotec). Biotinylated goat anti-rabbit immunoglobulins for immunohistochemistry were from Oncogen Research. Secondary antibodies for immunoblotting and fluorescence were from Jacksons Immunoresearch.

Gel electrophoresis and immunoblotting

Proteins were separated on $10 \%$ or $12 \%$ SDS-gels by polyacrylamide gel electrophoresis (PAGE) and electrotransferred onto nitrocellulose filters. Optical density measurements of the protein bands in immunoblots were performed with the BIO-PROFIL Bio-1D Windows Application V99.01. Protein content in XLAP2 bands was normalized according to the actin content in each lane.
Isolation of Xenopus egg extract

Egg collection and extract preparation were carried out according to Salpingidou et al. (2008).

Extraction of XLAP2 from cell nuclei isolated from XTC cells

XTC cells that had been cultured for $72 \mathrm{~h}$ were trypsinized, pelleted at $800 \mathrm{~g}$ and washed briefly with ice-cold phosphate-buffered saline (PBS). All preparation steps were performed at $4^{\circ} \mathrm{C}$. The cell pellet was homogenized in $2 \mathrm{ml}$ isotonic buffer consisting of $10 \mathrm{mM}$ TRIS-HCl pH $7.5,5 \mathrm{mM} \mathrm{MgCl}_{2}, 50 \mathrm{mM} \mathrm{NaCl}, 250 \mathrm{mM}$ sucrose, $0.1 \mathrm{mM}$ phenylmethane sulphonylfluoride (PMSF) and $10 \mu \mathrm{l} / \mathrm{ml}$ inhibitor cocktail (Sigma) in a glass Potter homogenizer with a tight pestle and the nuclei were recovered at $2000 \mathrm{~g}$. Equivalents of $6.6 \times \times 10^{6}$ nuclei were extracted for $20 \mathrm{~min}$ with $200 \mu \mathrm{l}$ of a solution consisting of $10 \mathrm{mM}$ TRIS-HCl buffer $\mathrm{pH} 7.5$ or of $10 \mathrm{mM}$ TRIS-HCl buffer $\mathrm{pH} 7.5$ containing $1 \%$ (vol/vol) Triton X-100, $1 \mathrm{M} \mathrm{NaCl}, 1 \%$ (vol/ vol) Triton X-100 plus $1 \mathrm{M} \mathrm{NaCl}$, or $6 \mathrm{M}$ Urea. Samples were fractionated by centrifugation at $10,000 \mathrm{~g}$ for $10 \mathrm{~min}$ into supernatant and pellet. Supernatants were supplemented with $50 \mu \mathrm{l}$ SDS sample buffer, whereas pellets were homogenized in $150 \mu \mathrm{l}$ SDS sample buffer and analysed by Western blotting. Of the supernatants and pellets, $10 \%$ were resolved on SDS-PAGE gel, transferred onto nitrocellulose filter and probed for XLAP2 protein.

Immuno-isolation of XLAP2 isoforms from Xenopus XTC cells and tissues

Frozen Xenopus liver pieces were powdered at liquidnitrogen temperature and incubated on ice with a lysis buffer consisting of PBS pH 7.4 containing $0.05 \%$ Tween $20,1 \mathrm{mM}$ dithiothreitole (DTT), $0.5 \mathrm{mM} \mathrm{MgCl}$, $0.2 \mathrm{mM}$ PMSF and $10 \mu \mathrm{l} / \mathrm{ml}$ inhibitor cocktail (Sigma). After sonication, the lysate was cleared of cell debris by centrifugation at $12,000 \mathrm{~g}$. The resulting supernatant was supplemented with 5\% SDS and $20 \mathrm{mM}$ DTT and heatdenatured. Proteins were precipitated with $10 \%$ trichloroacetic acid. The precipitate was then resuspended in immunoprecipitation buffer (Rzepecki and Fisher 2000) and used for protein isolation. Xenopus XTC tissue-cultured cells, $1.5 \times 0^{7}$ per test, were pelleted at $800 \mathrm{~g}$, washed, resuspended in lysis buffer and treated as above. The lowspeed supernatant fraction from Xenopus eggs was incubated on ice with gentle inversion after addition of $1 \%$ Triton X-100, 0.02\% SDS, 0.3 M NaCl, 0.2 mM PMSF and $10 \mu \mathrm{l} / \mathrm{ml}$ inhibitor cocktail. Centrifugation at $17,000 \mathrm{~g}, 4^{\circ} \mathrm{C}$, was carried out to produce a supernatant for immunoprecipitation. Immuno-isolation with specific affinity-purified 
anti-XLAP2 immunoglobulins, immobilized on Protein A Agarose (Sigma), was as described previously (Rzepecki and Fisher 2000).

For mass spectrometry, proteins immuno-isolated from eggs were resolved by "clean" SDS-PAGE and the gels were silver-stained. Gel fragments were excised from the gels in the region containing the protein band of interest and the samples were subjected to LC/MS/MS analysis.

\section{Microscopic procedures}

XTC cells were grown on coverslips, fixed with $4 \%$ paraformaldehyde in PBS and permeabilized with $0.5 \%$ Triton X-100 in PBS or $40 \mu \mathrm{g} / \mathrm{ml}$ digitonin in PBS. Small pieces of Xenopus tissues and staged embryos were fixed with 4\% paraformaldehyde in PBS containing $0.5 \%$ Triton $\mathrm{X}-100$ and paraffin-embedded. Tissue sections were prepared for immunohistochemistry and immunofluorescence. For imaging, two fluorescence microscopes were used: a confocal microscope LSM510 META with an FCS system (Zeiss) and an Olympus IX70 fluorescence microscope with a two-channel confocal laser scanning unit (FV500). Any brightness and contrast adjustments were performed in Adobe Photoshop or Zen 2007 (Zeiss).

\section{Electron microscopy}

Transmission electron microscopy (TEM) and immunogold labelling was as described previously (Salpingidou et al. 2008). Affinity-purified anti-XLAP2 IgGs were used for the immunogold staining of tissues, cultured cells and NEs. Images of somatic tissues were taken via a Jeol B 100 transmission electron microscope at magnifications of $\times 20,000$ and $\times 40,000$. XTC cells were either embedded in Araldite (Agar Sciences) for TEM or prepared for cryosectioning and immunogold labelling. XTC samples were examined under a Hitachi H7600 transmission electron microscope.

For scanning electron microscopy (SEM), oocytes were dissected out from hormone-stimulated female frogs. The nuclei were manually isolated, placed on silicone chips and processed for immunogold labelling as described by Allen et al. (2007). SEM was performed on Hitachi S-5200.

Statistical analyses of clusters immunogold-labelled NEs of Xenopus oocytes examined by SEM

From each micrograph, immunogold label coordinates were digitalized by using Scion Image software and saved as data files with two variables ( $\mathrm{X}$ and $\mathrm{Y}$ coordinates). The left, right, top and bottom boundaries were subsequently determined (maximum and minimum of coordinate variables) and another data set with the same number of observations was created with the $\mathrm{X}$ and $\mathrm{Y}$ coordinates being randomly assigned values from the uniform distribution and within the same boundaries as in the original file. The basic logic behind this methodology can be described as follows. If the tendency of empirical (observed) points to form clusters is more likely than that of points with randomly assigned coordinates (theoretical points), then we can say that a general clustering tendency exists in the observed data. The major obstacle in statistical analyses is that the LAP2 signal is excluded from the nuclear pore complex (NPC) area, narrowing the space for LAP2. We overcame this problem by introducing the same distance values between labels in the control as in the original micrograph. Data were analysed with SPSS 17 software by three methods: hierarchical clustering, K-means method and Bacher's method. The statistical significance of the cluster analyses was calculated by using the following tests: the one-sided two-sample $t$-test for means, the non-parametric Z-test (Kolmogorov-Smirnov test) and the U-test (Mann-Whitney). Statistical significance was assumed at values of $P<0.05$.

Statistical analyses of immunogold labelling of Xenopus XTC cells examined by TEM

The calculated relative labelling intensity for XLAP $2 \beta$ in four different regions inside the cell nucleus of XTC cells was based on cryosectioned and immunogold-labelled XTC cells according to the method described in Mayhew (2005). XTC cells were cryosectioned and immunogold-labelled; 517 immunogold labels were counted from eight micrographs at the same magnification. The standard deviation error for the relative labelling intensity (RLI) was calculated by Student's $t$-test and was $P<0.05$ for all regions. The areas of the entire nucleus, the $\mathrm{NE}$ and the nuclear invagination were calculated from the middle Z-section of confocal microscope images. The areas of the remaining two regions were calculated proportionally also from the middle Zsection of the confocal microscope image by assigning them the remaining nucleus area after the subtraction of the $\mathrm{NE}$ and invaginations areas. For the calculations of areas from confocal images, we used Zen 2008 software for the Zeiss LSM 510 confocal microscope.

\section{Results}

Characterization of antibodies and identification of 40-kDa polypeptide as XLAP2 $\gamma$ isoform by using LC/MS/MS method

We generated rabbit antibodies against the common $\mathrm{N}$ terminal 1-165-aa fragment of XLAP2 protein. The specific 
immunoglobulins were affinity-purified by using the XLAP2 N-terminal fragment (Salpingidou et al. 2008; Supplemental Fig. 1). With this antibody, the previously described XLAP $2 \beta$ protein $(66 \mathrm{kDa})$ was immunoprecipitated from Xenopus liver and XTC cultured cells, as confirmed by Western blot (not shown).

In order to identify additional XLAP2 polypeptides and link them to known cDNAs, we employed immunoprecipitation and mass spectrometry (LC/MS/MS). Proteins were first immunoprecipitated from Xenopus egg extracts by using the XLAP2 antibody. These proteins were immunoblotted with anti-XLAP2 (Fig. 1 a) to detect possible LAP2 isoforms. Two polypeptides of $86 \mathrm{kDa}$ and $40 \mathrm{kDa}$ were consistently detected. Gel fragments containing these two proteins (Fig. 1b) were further analysed by LC/MS/MS. Our mass spectrometry data identified an $86-\mathrm{kDa}$ polypeptide as clone $2 / \omega$ and the $40-\mathrm{kDa}$ polypeptide as a translation product of a previously uncharacterized cDNA (see Discussion, Fig. 1c). According to the existing LAP2 nomenclature, the $40-\mathrm{kDa}$ isoform should be named LAP $2 \gamma$. The identified LAP2 $\gamma$ peptide is not a degradation product of XLAP $2 \omega$, since solubility assays have confirmed that this protein contains the transmembrane domain, which is located on the C-terminal end of XLAP2 $\gamma$ (and of XLAP2 $\omega$ ). Our antibodies are N-terminus-specific and so the potential degradation product of that size would not be recognized by our antibodies because of the cleaved off N-terminal part of the protein with all epitopes. Moreover, the $40-\mathrm{kDa}$ protein possesses a transmembrane domain and reacts with LEM-domain antibodies, as has been described previously (Lang et al. 1999) in Xenopus oocytes and unfertilized eggs.

Notably, the clone 3 cDNA, which is the only known candidate for coding the XLAP2 $\gamma$ protein, lacks the TM domain, exon $\mathrm{C}$ and the lamin-binding region characteristic for major human LAP2 proteins. This confirms that it cannot be translated into XLAP2 $\gamma$ protein.

XLAP $2 \omega$ and XLAP $2 \gamma$ are expressed during early stages of development, whereas XLAP $2 \beta$ is expressed late in development and in adult tissues

The Western blot analysis of XLAP2 protein levels during development demonstrated the presence of at least three differentially expressed isoforms (Fig. 2a). XLAP $2 \omega$ and XLAP2 $\gamma$ occur in eggs and are expressed during development up to stage 41 with the maximum around stage 20. The continuous presence of XLAP2 $\gamma$ along with XLAP $2 \omega$ is notable. Expression of the $66-\mathrm{kDa}$ XLAP2 $\beta$ isoform is only observed from the neurula stage onwards and its expression levels reach a maximum from stage 34. In order to confirm an earlier observation that XLAP $2 \beta$ is the sole isoform after stage 41 , we analysed
Fig. 2 Developmentally regulated expression and subcellular localization of XLAP2 isoforms in Xenopus. a Immunoblot analysis of XLAP2 proteins during development. Positions of three major protein bands representing XLAP2 isoforms are marked together with an actin band used as a loading control. The embryonic XLAP2 proteins, viz. XLAP $2 \omega$ and XLAP $2 \gamma$, are the only two isoforms present up to gastrula stage, showing an accumulation of the amount of protein until stage 22 followed by a steady decrease up to stage 41 and its disappearance from stage 44 (a, right). Expression of the somatic XLAP2 $\beta$ isoform is first detected at the gastrula stage and increases significantly from the 28th stage. The additional protein band of $76 \mathrm{kDa}$ may be a degradation product of the $86-\mathrm{kDa}$ isoform. Molecular masses of reference proteins (in $\mathrm{kDa}$ ) are marked. b-f Subcellular localization of XLAP2 protein isoforms during Xenopus development and in XTC cells. The paraffin sections from Xenopus embryos and adult tissues were probed for XLAP2 $(\mathbf{b}, \mathbf{c})$ or costained for XLAP2 (red) and either lamin B2/B3 (d) or lamin B2 (e, f; green). DNA was stained with 4,6-diamidino-2-phenylindole (DAPI; blue). Images were via a fluorescent (b) or confocal laser scanning (c-f) microscope. Images represent the Z-stack (c) or single Z-section (b', d-f; $0.25 \mu \mathrm{m}$ thick). b During early development, XLAP2 proteins colocalize with mitotic chromosomes in the morula and blastula in which XLAP-86 and XLAP2-40 are expressed and with the interphase nuclei of the gastrula and stage-20 embryos in which XLAP-86, XLAP2-66 and XLAP2-40 are present (arrowheads positions of cell nuclei, arrow position of XLAP2). Inset top right of morula merged image in $\mathbf{b}$ Higher magnification of the boxed nucleus. The localization of XLAP2 in the nuclear envelope (NE) is rare in blastula

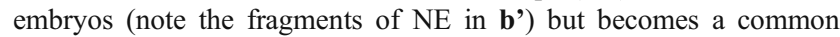
feature at the gastrula stage (see well-organized membranes in $\mathbf{b}^{\prime}$ ). Embryonic samples show autofluorescence (mostly from the yolk platelets) that reveals the shape and size of each cell (e.g. easily seen in red and blue channels from morula to gastrula stage). c In the morula stage embryos, XLAP2-containing fractions are associated with mitotic chromosomes (c.1) and karyomere formation is seen around individual chromosomes (c.2). Note the position of embryonic XLAP2 in membrane-like structures on the morula chromatin (arrow), the typical cluster-like location on the chromatin (arrowhead) and the XLAP2 cluster outside the chromatin (double arrowhead). In somatic cells, XLAP2 $\beta$ aggregates in between separating chromatids at early anaphase (c.3) and then associates with peripheral regions of chromatin in late anaphase (c.4; arrows peripheral regions of chromatids with associated XLAP2, arrowheads chromosome core regions not yet associated with XLAP2). d-f The XLAP2 proteins colocalize with B-type lamins in stage 26-28 (d) and stage 44 (e) Xenopus embryos and in adult tissues (f). Images represent brain tissue. Bars $100 \mu \mathrm{m}$ (b, morula), $20 \mu \mathrm{m}$ (b, blastula - stage 20), $5 \mu \mathrm{m}$ (c), $20 \mu \mathrm{m}(\mathbf{d}), 10 \mu \mathrm{m}\left(\mathbf{b}^{\prime}, \mathbf{e}, \mathbf{f}\right)$

stages 42 up to 52 by using a chemiluminescent method to increase the sensitivity of Western blot detection (Fig. 2a, black and white section). This experiment confirmed that XLAP $2 \omega$ and XLAP2 $\gamma$ were not present in later developmental stages.

\section{Subcellular localization of XLAP2 proteins} during development and in adult tissues

Immunofluorescence analyses indicated that embryonic XLAP2 proteins ( $\omega$ and $\gamma$ ) localized to the chromatin throughout the cell cycle during early development (up to the gastrula stage). At the morula stage, a significant 
a

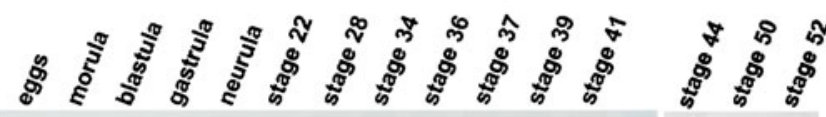

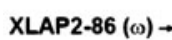

XLAP2-66 ( $(\beta) \rightarrow$

XLAP2-40(y)

b

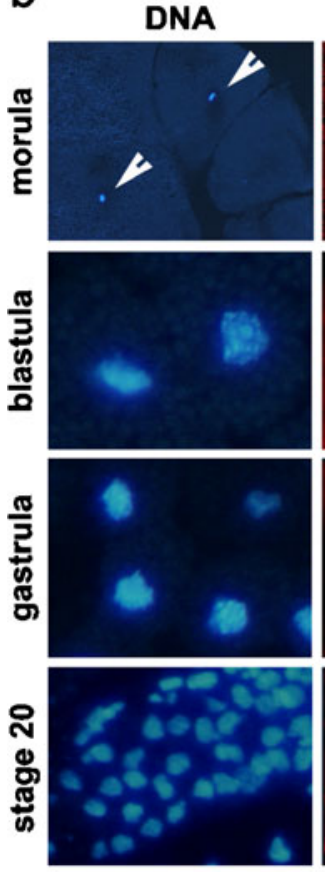

XLAP2
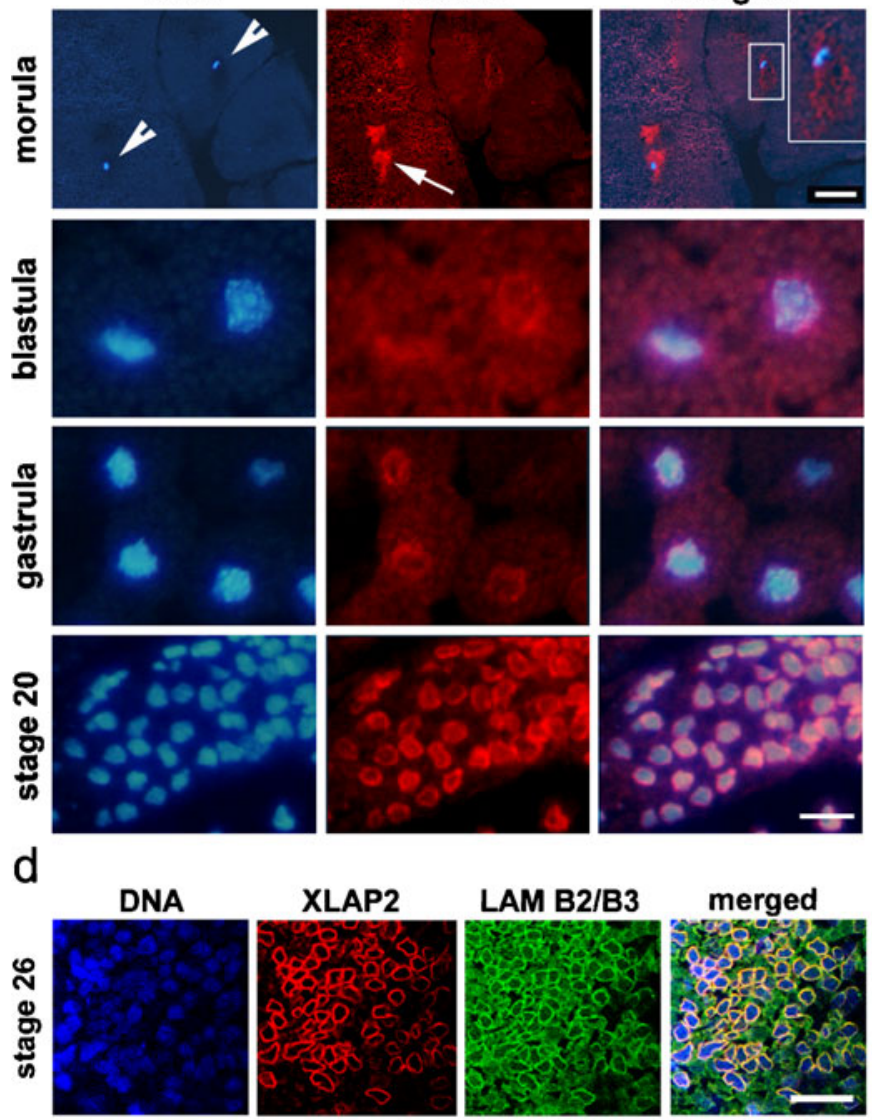

e
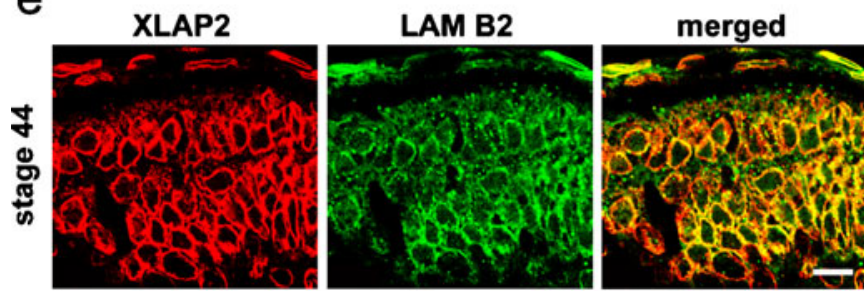

amount of XLAP2 was located around the chromatin, frequently forming cloud-like structures inside the cell nucleus (Fig. 2b, arrow). XLAP2 was distributed irregularly but in an NE-like fashion in the cell nucleus (Fig. 2b, inset). At later stages of development, both embryonic XLAP2 isoforms were located typically at the NE, as easily visible at stage 20 (Fig. 2b) and through later

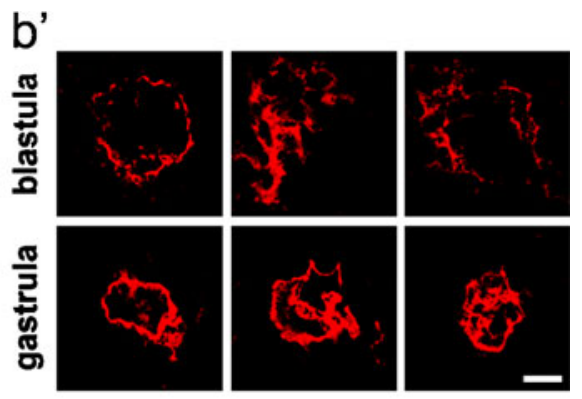

C
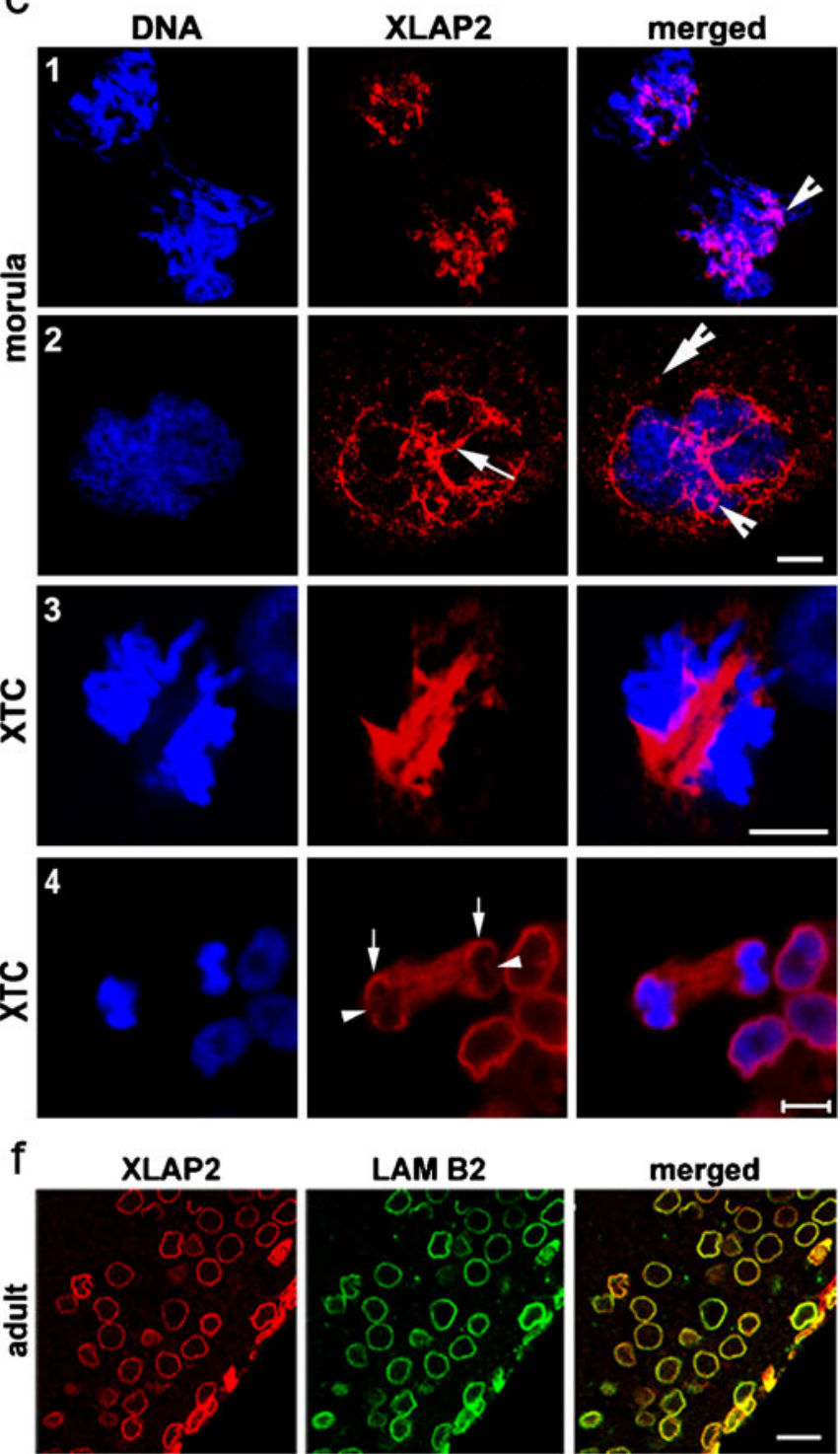

stages (Fig. 2d, e). Irregular but complete NEs were noticeable at the blastula stage, with regular NEs at the gastrula stage (Fig. 2b'). Detailed immunofluorescence studies revealed that both embryonic XLAP2 isoforms were associated with karyomers in interphase nuclei at the morula stage. Mitotic chromatin was also associated with embryonic XLAP2 proteins (Fig. 2c, 1st anaphase 
and 2nd interphase respectively). From the blastula stage, both embryonic XLAP2 proteins were localized to the NEs that had formed around the interphase chromatin. The amounts of embryonic XLAP2 proteins diminished during development but they still associated with mitotic chromatin. From stage 20, XLAP $2 \omega$ and XLAP2 $\gamma$ localized generally to the NEs and colocalized with lamins B2/B3 and lamin B2 in all tissues tested (e.g. brain, ectoderm and somites; not shown). As demonstrated in Fig. 2d-f, XLAP2 proteins occurred in the brains of stage-26 and stage-44 embryos (Fig. 2d, e, respectively) and in adult Xenopus (Fig. 2f). During early anaphase in XTC tissue-cultured cells, XLAP2 $\beta$ was localized in between the chromatids and, in late anaphase, associated with the chromatin (Figs. 2c, 3, 4). The association of XLAP2 $\beta$ with peripheral chromatin was readily visible (Figs. 2c, arrows), whereas internal regions were still weakly bound by XLAP2 $\beta$ (Figs. 2c, arrowheads). Detailed immunofluorescence analyses of the cell-cycle-dependent distribution of the XLAP2 $\beta$ isoform in XTC cells also revealed complete colocalization with lamin B2 (Supplemental Fig. 2). Punctate labelling of NPCs with m414 antibodies (anti-Nup62 and other $\mathrm{F} / \mathrm{G}$ repeat nucleoporins) overlapped with the smooth continuous LAP2 labelling of NE at interphase (Supplemental Fig. 2).

XLAP2 $\beta$ is localized to the inner nuclear membrane of NE but also to chromatin inside the cell nucleus and to NE invaginations

XLAP2 $\beta$ was ubiquitously expressed in all adult tissues tested but was absent from oocytes and eggs in which the $\omega$ and $\gamma$ isoforms were expressed (see Supplemental Fig. 1b). The location of the XLAP $2 \beta$ protein was demonstrated in the brain and in muscle cells by TEM after immunogold labelling in Fig. 3a, b. XLAP2 $\beta$ was located mostly in heterochromatin regions next to the NE. Nevertheless, a small fraction of the protein (typically between $10 \%$ and $30 \%$ of the total label) was detected within chromatin microdomains inside the nucleus. This interpretation was supported by the immunofluorescence confocal microscopy data from brain tissue (Fig 2f) in which XLAP2 $\beta$ was located predominantly in the NE of eppendymocytes and glial cells (see also Supplemental Fig. 1e). As illustrated in Fig. 3b, immunogold labelling of muscle and muscle satellite cells nuclei demonstrated the labelling of the heterochromatin regions next to the NE and at some distance from the NE. Solubility assays on XTC cells (Fig. 3e) and on liver and brain tissues (not shown) indicated that the only protein detected $(66 \mathrm{kDa})$ behaved as an integral membrane protein suggesting that the fraction of XLAP $2 \beta$ inside the nucleus possessed a transmembrane domain.
In order to solve the question of the XLAP $2 \beta$ presence in the nuclear interior, we performed immunogold labelling on tissue-cultured XTC cells serving as a model of somatic tissues. Immunogold labelling indicated that XLAP2 $\beta$ was associated with heterochromatin adjacent to the NE (Fig. 3f-j, arrowheads), although some clusters of XLAP2 protein were also present in the heterochromatin inside the nucleus (Fig. 3f-j, double arrowheads). Only a few of them were accompanied by membranes of NE invaginations in osmium-stained cryosections from XTC cells (see also Supplemental Fig. 3f demonstrating a cluster of XLAP2 protein on chromatin inside the nucleus and its location in relation to NE membranes). In parallel, we performed confocal microscopy on XTC cells; this confirmed that the predominant fraction of XLAP2 was associated with the NE but that a small fraction of the protein was also detected inside the nucleus (see the three strong signals in central Zsections of Fig. 3d). These particular intra-nuclear signals probably represented long invaginations of the NE, because the signal was present in consecutive $\mathrm{Z}$ sections beginning from the NE and nuclear periphery to the centre of the nucleus. Thus, at least part of intra-nuclear XLAP2 protein was associated with NE invaginations. A small fraction of XLAP $2 \beta$ protein, presumably associated with intra-nuclear chromatin, seemed to be also dispersed throughout the interior of the cell nucleus. This particular fraction, which could be visualized at early prophase, was located between the condensing chromatids (see Fig. 3c, arrowheads).

We further investigated XLAP2 $\beta$ locations by using statistical analyses. We analysed the distribution of immunogold-labelled XLAP2 $\beta$ protein in cryosections from XTC cells by using TEM. We examined the distribution of immunogold label ( $\mathrm{n}=517$ spots) from eight micrographs, divided them into four groups and calculated the RLI compared with the control, i.e. the randomly generated labelling of the entire cell nucleus (see Materials and methods). The chosen regions were: chromatin at the $\mathrm{NE}$, chromatin inside the nucleus associated with the NE, chromatin inside the nucleus not associated with NE, and the nuclear interior with no chromatin and no NE. Table 1 reveals that the XLAP2 $\beta$ distribution is not random and is always associated with chromatin. However, a significant portion of protein is associated with chromatin but is distant from the NE, inconsistent with a location in the membrane. Moreover, $72 \%$ of the calculated label in the first three groups is found in clusters of four or more labels suggesting a microdomain-like distribution of XLAP $2 \beta$ both at the $\mathrm{NE}$ and at the intra-nuclear chromatin. In order to support this finding, we used confocal microscopy. We measured the intensity of the fluorescent XLAP2-specific signal in $0.5-\mu \mathrm{m}$-thick optical sections through the centre of five nuclei. The calculated relative intensities of the fluorescent signal from NE, from NE invaginations and 

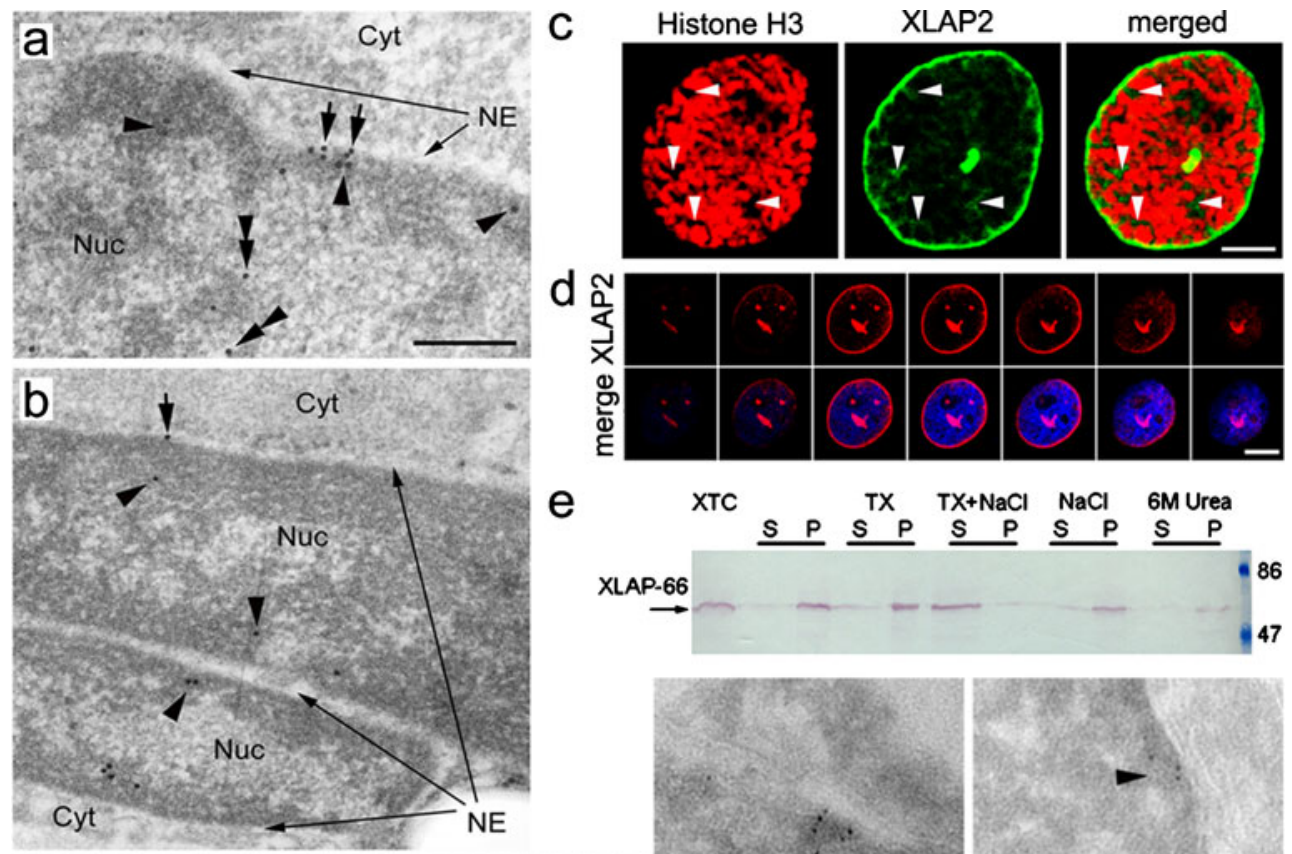

e
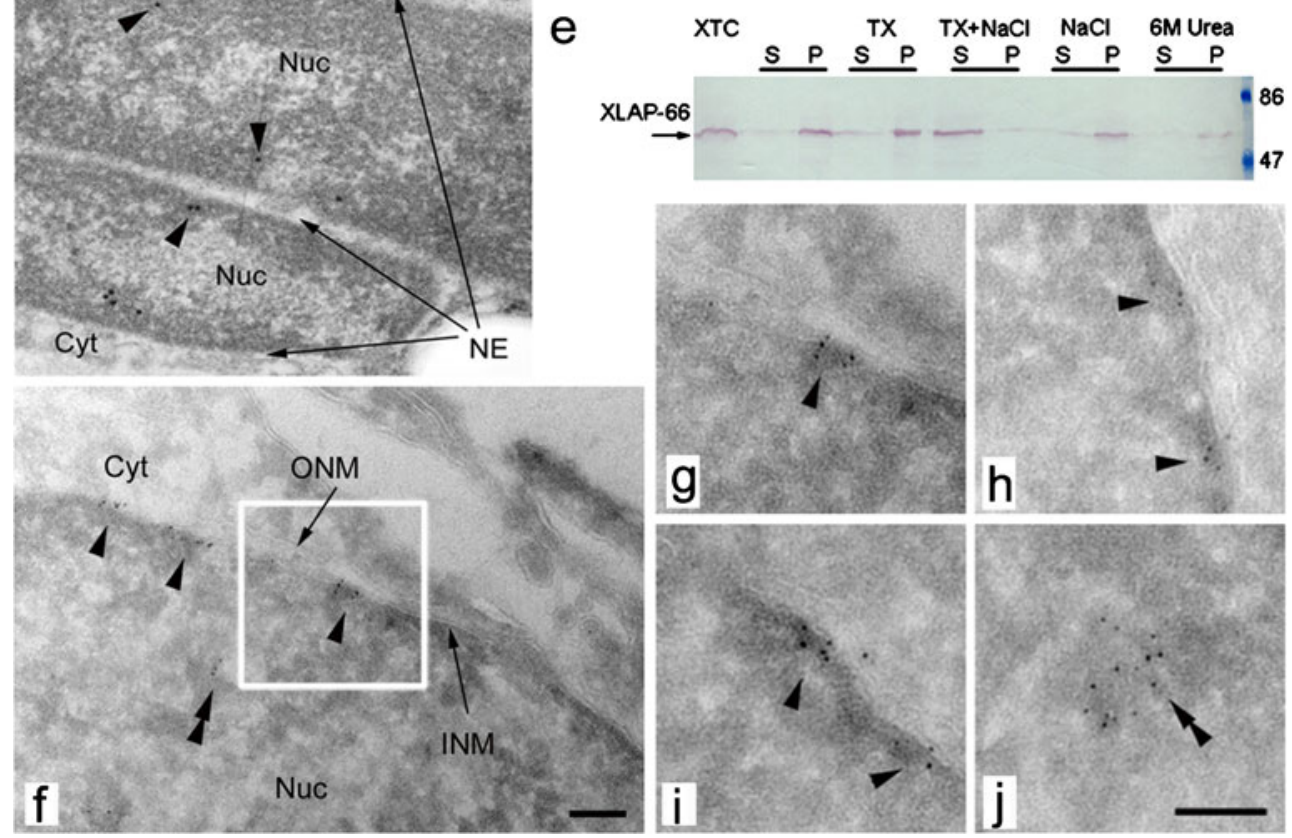

Fig. 3 Subcellular localization of somatic XLAP2 protein in adult tissues and XTC cultured cells. a, b Immuno-gold staining of ultrathin sections of embedded tissues with antibodies against XLAP2, followed by incubation with protein A conjugated to 8-nm gold particles (Cyt cytoplasm, $N E$ nuclear envelope, $N u c$ nucleus). In brain (a) and muscle (b) tissues, XLAP2 (black dots) localizes mostly at the inner nuclear membrane (arrows) and peripheral heterochromatin (arrowheads) but is also present in intra-nuclear regions (double arrowheads). b Note that XLAP2 occurs both in the nucleus of the myotube (top) and in the muscle satellite cell (bottom). Bar $500 \mathrm{~nm}$. c In early prophase of XTC cells, costaining for XLAP2 (green) and phospho-histone $\mathrm{H} 3$ (red) reveals that XLAP2 $\beta$ is located not only at the NE, but also in intra-nuclear loci (arrows). Bar $5 \mu \mathrm{m}$. d Confocal studies of interphase XTC tissue cultured cells stained for XLAP2 demonstrate that XLAP $2 \beta$ is present in NE and in invaginations of the nuclear membrane (strong dots inside the nucleus). Note that the region lying on the upper left side of the nucleus corresponds to the nucleolus area (see merge). Bar $10 \mu \mathrm{m}$. e Biochemical properties of XLAP2 $\beta$ protein from XTC tissue-cultured cells. Analysis of the
XLAP2 $\beta$ polypeptide solubility in isolated cell nuclei, extracted with $10 \mathrm{mM}$ TRIS buffer alone or containing Triton X-100 (TX), NaCl, Triton X-100 plus $\mathrm{NaCl}(\mathrm{TX}+\mathrm{NaCl})$, or urea (6M Urea). Equivalent amounts of the supernatants and pellets were resolved in SDS-PAGE gel, transferred onto nitrocellulose filter and probed for XLAP2 protein. XLAP2-66 displayed the properties of an integral membrane protein involved in interactions with other protein components of the nuclear lamina, because it was solubilized in the presence of Triton X100 together with $\mathrm{NaCl}$. $\mathbf{f}-\mathbf{j}$ Transmission electron microscopy (TEM) studies of XTC tissue-cultured cells confirm the NE localization for XLAP2 proteins. XTC cells were grown in culture, embedded in gelatin and cryo-sectioned for immuno-gold TEM. XLAP2 was detected with antibodies against XLAP2 followed by Protein A coupled with 5-nm gold particles. Gold-labelled XLAP2 was mostly localized at the nuclear periphery and inner nuclear membrane (INM, arrowheads) but intra-nuclear clusters (double arrowheads) were also present $(\mathbf{f}, \mathbf{j})$. g Higher magnification of the boxed area in $\mathbf{f}(\mathrm{Cyt}$ cytosol, ONM outer nuclear membranes, Nuc nucleus). Bars $100 \mathrm{~nm}$

typical invagination with a visible lumen (Fig. 4a, arrow) and other structures without a visible lumen (Fig. 4a, arrowheads). XLAP2 $\beta$ in the NE, at the upper and the lower part of cell nucleus, was not homogeneously distributed (Fig. 4b). This could represent a cluster-like from the nuclear interior were $165 \pm 11,159 \pm 16,45 \pm 14$, respectively. This correlated with the calculated RLI values from TEM micrographs.

Two types of structures containing XLAP $2 \beta$ and lamin B2 were present inside the cell nuclei of XTC cells: a 

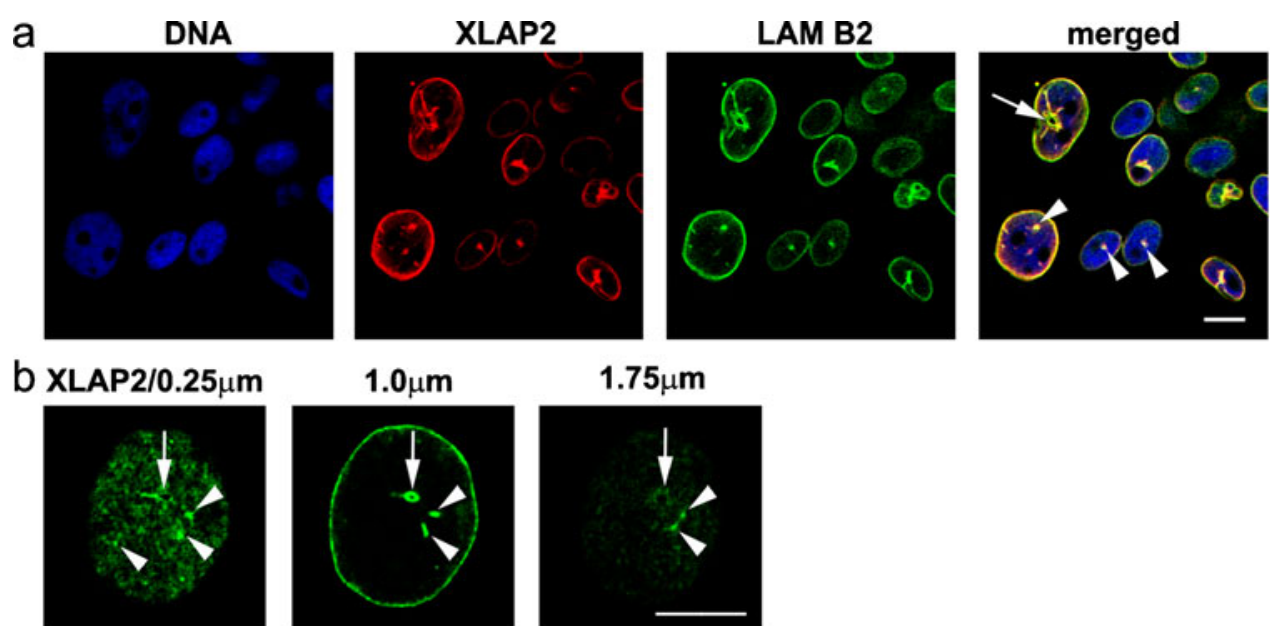

$1.0 \mu \mathrm{m}$

$1.75 \mu \mathrm{m}$
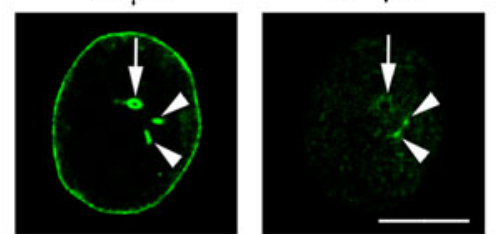

C

DNA

XLAP2
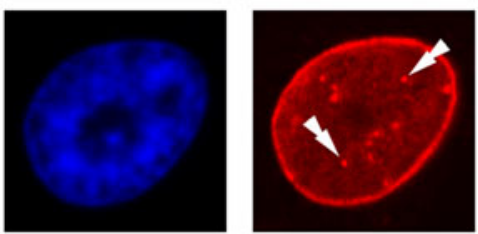

LAM B2

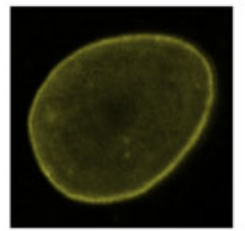

DHCC

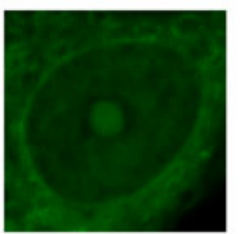

merged
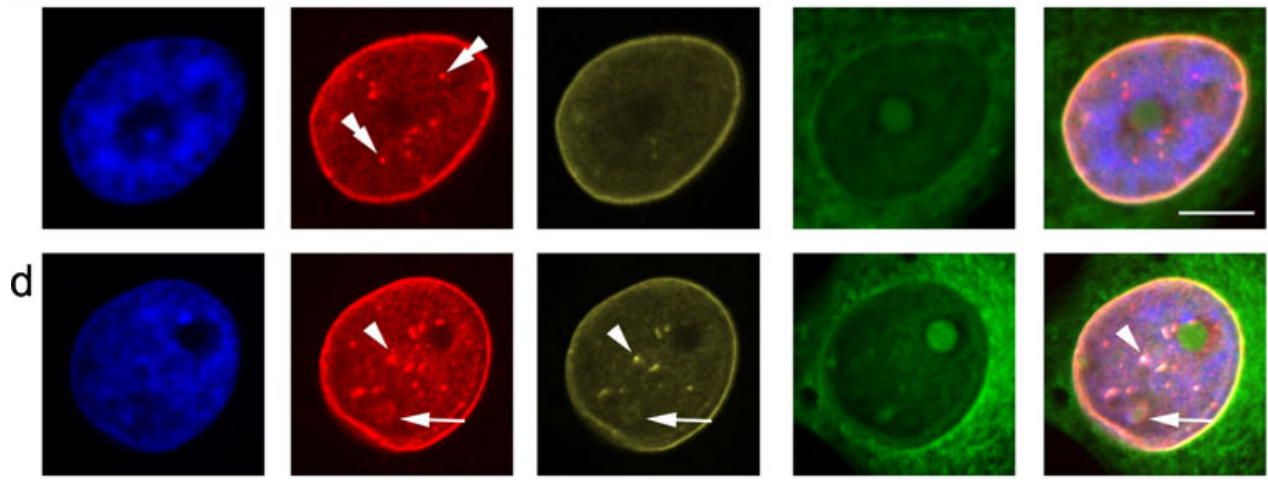

e

DNA

XLAP2
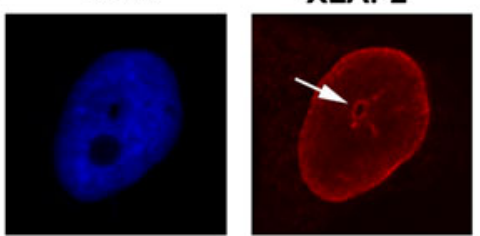

DHCC

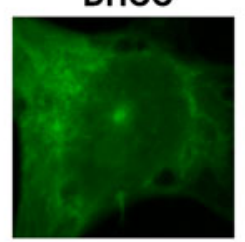

merged

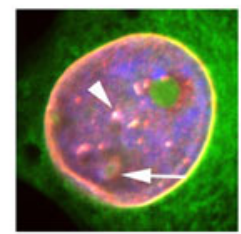

f

XLAP2

p62-Nup F/G
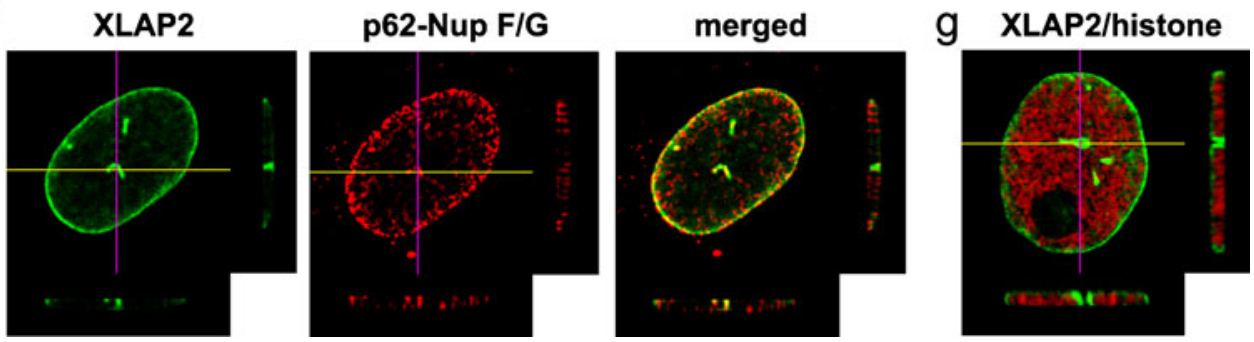

structure containing XLAP2 at the NE. Some of such structures spanned throughout the cell nucleus (e.g. typical invagination and "non-lumenal" structures, Fig. 4b, arrows and arrowheads, respectively). In all invaginations with a visible lumen, XLAP2 colocalized with lamin B2 (Fig. 4d); the lumenal part was free of DNA (Fig. 4d, e) and stained with dihydrocholecalciferol (Fig. 4e) indicating the endoplasmic-reticulum-like structures present inside.
Three-dimensional reconstructions of series of confocal images of a single nucleus (Fig. 4f, g) confirmed that such lumenal structures connected opposite sides of the cell nucleus membrane, that they were free of DNA (Fig. 4g) but that they contained F/G-repeat nucleoporins (Fig. 4f) suggesting the presence of NPCs on such structures. Only a few out of the many internal non-lumenal structures containing XLAP2 also contained lamin B2 (Fig. 4c, d). 
4 Fig. 4 Intra-nuclear localization of XLAP2 as correlated to nuclear antigens and lipid membranes. XTC tissue-cultured cells were fixed with paraformaldehyde and probed for XLAP2 (green in $\mathbf{b}$, red in e) or costained for XLAP2 (red in a, c, d) and lamin B2 (LAM B2, green in $\mathbf{a}$, yellow in $\mathbf{c}, \mathbf{d}$ ) or costained for XLAP2 (green, $\mathbf{f}, \mathbf{g})$ and either p62-F/G nucleoporins ( $p 62-N u p F / G$, Ab 414, red, f) or phosphorylated histone $\mathrm{H} 3$ ( $r e d$, g). Cellular membranes were visualized with dihydrocholecalciferol (DHCC) and DNA was counterstained with DAPI. a Various phenotypes of cultured cells presenting large nuclear channels (arrow) or nuclear dots (arrowheads) in which XLAP2 colocalizes with lamin B2. b Large channels (arrows) and smaller invaginations (arrowheads) are present on consecutive Z-sections of the cell nucleus from the bottom to the top. c, d XLAP2 staining correlates with lamin B2 and lipid membranes in NE. The nuclear territory contains nuclear dots/invaginations that colocalize with lamin B2 (arrowheads) or show a single XLAP2 signal (double arrowheads), forming channels (arrow) concomitant with the membrane signal (DHCC). e Cell with a large nuclear channel lacking DNA but possessing membrane signal (arrow). f, $\mathbf{g}$ Large nuclear invaginations or channels running from the bottom to the top of a nucleus. Images represent single $\mathrm{Z}$-section $(0.25 \mu \mathrm{m}$ thick) from the nucleus, with the lines marking the surface of the $\mathrm{Z}$-axis cross section through the nucleus. The cross sections from seven consecutive Z-stacks are shown bottom and right. Note that XLAP2 signal colocalizes with $\mathrm{p} 62-\mathrm{F} / \mathrm{G}$ repeats containing nucleoporins (f) or with branches of the DNA-free tunnel in the nucleus (g). Bars $10 \mu \mathrm{m}(\mathbf{a}, \mathbf{b}, \mathbf{e})$, $5 \mu \mathrm{m}(\mathbf{c}, \mathbf{d})$

This could represent the internal chromatin-bound clusters of XLAP2 detected in TEM experiments.

Embryonic XLAP2 isoforms locate at microdomains at the inner nuclear membrane of oocyte NE

Analyses of the distribution of embryonic XLAP2 isoforms were performed by examining manually isolated oocyte nuclei with SEM following immunogold labelling. XLAP2 $\omega$ and XLAP2 $\gamma$ were located at the inner NE membrane outside of the NPCs (Fig. 5a, b, yellow dots). This was in agreement with the detailed analyses of TEM immunogold data from adult tissues and XTC cells in which XLAP2 $\beta$ location was not associated with NPCs (see Fig. 3a,b, f-j). The distribution of embryonic XLAP2 isoforms was not uniform (see Fig. 5c, d) and could reflect the distribution of nuclear lamina filaments underlying the inner nuclear membrane of the NE.

We performed hierarchical analyses of clusters based on the Euklidean distance between the label and single linkage method followed by a calculation of the potential cluster number by using the dendrogram method, on the basis of at least three labels as a potential cluster (see Materials and methods; see also Supplemental Fig. 2). Statistical analyses of 356 immunogold labels from six micrographs revealed the non-random distribution of the label with the Student's $t$-test value of $P<0.05$. Our statistical approach allowed us to identify the most probable number of clusters in each micrograph. The example presented in Supplemental Fig. 3a contains five clusters.

\section{Discussion}

The presence of several (up to five) isoforms of XLAP2 protein has long been proposed in Xenopus. Since the discovery of the first XLAP2 cDNA clones, several reports have suggested the existence of XLAP2 proteins $\beta$ and $\omega$ (Lang and Krohne 2003; Lang et al. 1999), and clones 2, 3 and 4 (Gant et al. 1999). Our mass spectrometry data (Fig. 1) have confirmed the identity of the XLAP2-86 polypeptide as $\mathrm{XLAP} 2 \omega /$ clone 2 and of the $40-\mathrm{kDa}$ polypeptide as XLAP2 $\gamma$ but without answering the question of its original cDNA. In addition to the mass spectrometry results, we have found that our IgG precipitates XLAP2 $\gamma$ from egg extract. XLAP2 $\gamma$ also fractionates with the MP2/NEP-B (NE vescicle population $\mathrm{B}$ ) fraction of membranes from the low-speed supernatant from eggs (Salpingidou et al. 2008) and its solubility when it is extracted from eggs and from the membrane fraction of eggs is identical to the solubility of XLAP2 $\omega$ and XLAP2 $\beta$ from XTC cells (Fig. 3).

The expression pattern of XLAP2 isoforms during Xenopus development as demonstrated in our experiments differs slightly from previous data (Lang et al. 1999). First of all, we demonstrate the presence of two (instead of one) early development-specific isoforms: XLAP $2 \omega$ and XLAP2 $\gamma$ (Fig. 2). In zebrafish, ZLAP2 $\omega$, the largest LAP2 isoform $(84 \mathrm{kDa})$, is the only previous embryonic isoform discovered (Schoft et al. 2003) and is also replaced late in development by the adult ZLAP2 $\beta$ isoform. Another difference is that, in Xenopus and zebrafish, all identified LAP2 proteins are integral membrane proteins, whereas in mammals, at least two LAP2 proteins are "soluble" nucleocytoplasmic proteins.

The embryonic XLAP2 isoforms ( $\omega$ and $\gamma$ ) are associated with chromatin and separated karyomers at the morula and blastula stage (Fig. 2c.1, c.2). This resembles the intra-cellular localization of zebrafish ZLAP2 $\omega$ protein observed in early embryos up to the late gastrula stage (Schoft et al. 2003) and suggests similar roles for embryonic LAP2 isoforms in lower vertebrates. The association of XLAP $2 \omega$ and $\gamma$ with mitotic chromatin decreases gradually during development up to late gastrula stage. Notably, XLAP2 $\beta$ appears at this particular stage of development and is mostly associated with the NE and NE-associated chromatin. This confirms the in vitro data from the native-gel shift assays, that bacterially expressed LAP2 cDNA clones $2(\omega), 3$ and $4(\beta)$ bind to BAF and chromatin with different affinities (Shumaker et al. 2001). Xenopus LAP2 isoforms have additional exons, designated $\mathrm{A}, \mathrm{B}$ and $\mathrm{C}$ (Fig. 1c), compared with mammalian LAP2s. XLAP2 $\beta$ cDNA lacks exon A. This may contribute to the different properties of the particular XLAP2 proteins in binding to chromatin (Shumaker et al. 2001). 
Table 1 Calculated relative labelling intensity $(R L I)$ for XLAP2 $\beta$ in $\mathrm{XTC}$ cells of four different regions inside the cell nucleus. XTC cells were cryosectioned and labelled as in Fig. 3f-j. Immunogold label $(n=517)$ was counted from eight micrographs at the same magnification. The standard deviation error for RLI was $P<0.05$ for all regions (Student's $t$-test). The area of the entire nucleus, nuclear envelope
$(N E)$ and nuclear invagination was calculated from the middle Zsection of the confocal microscope image from Fig.3c. The area of the remaining two regions was calculated proportionally also from the middle Z-section of the confocal microscope image after subtraction of the NE and invaginations ( $n / d$ not determined)

\begin{tabular}{lll}
\hline Region of the nucleus & RLI & Number of clusters detected (with 4 or more label) \\
\hline Chromatin at the NE & 19.33 & 18 \\
Chromatin inside nucleus with NE & 12.42 & 9 \\
Chromatin inside nucleus without NE & 7.96 & 12 \\
Nuclear interior with no chromatin and no NE & 0.086 & 2 \\
Random, statistical labelling of entire nuclear region & 1.00 & $\mathrm{n} / \mathrm{d}$ \\
\hline
\end{tabular}

Taken together, the XLAP $2 \omega /$ clone 2 and XLAP $2 \gamma$ isoform are expressed in early embryos and associate with interphase and mitotic chromatin, with the XLAP $2 \omega /$ clone 2 possessing the highest binding activity with BAF and chromatin compared with all isoforms examined; this might reflect the evolutionary adaptation to extremely rapid cell divisions at this stage of development. In contrast, XLAP $2 \beta$ expression starts when the cell divisions are slower and interphase is more pronounced, concomitant with the nine-fold lower affinity of binding with BAF (Shumaker et al. 2001).
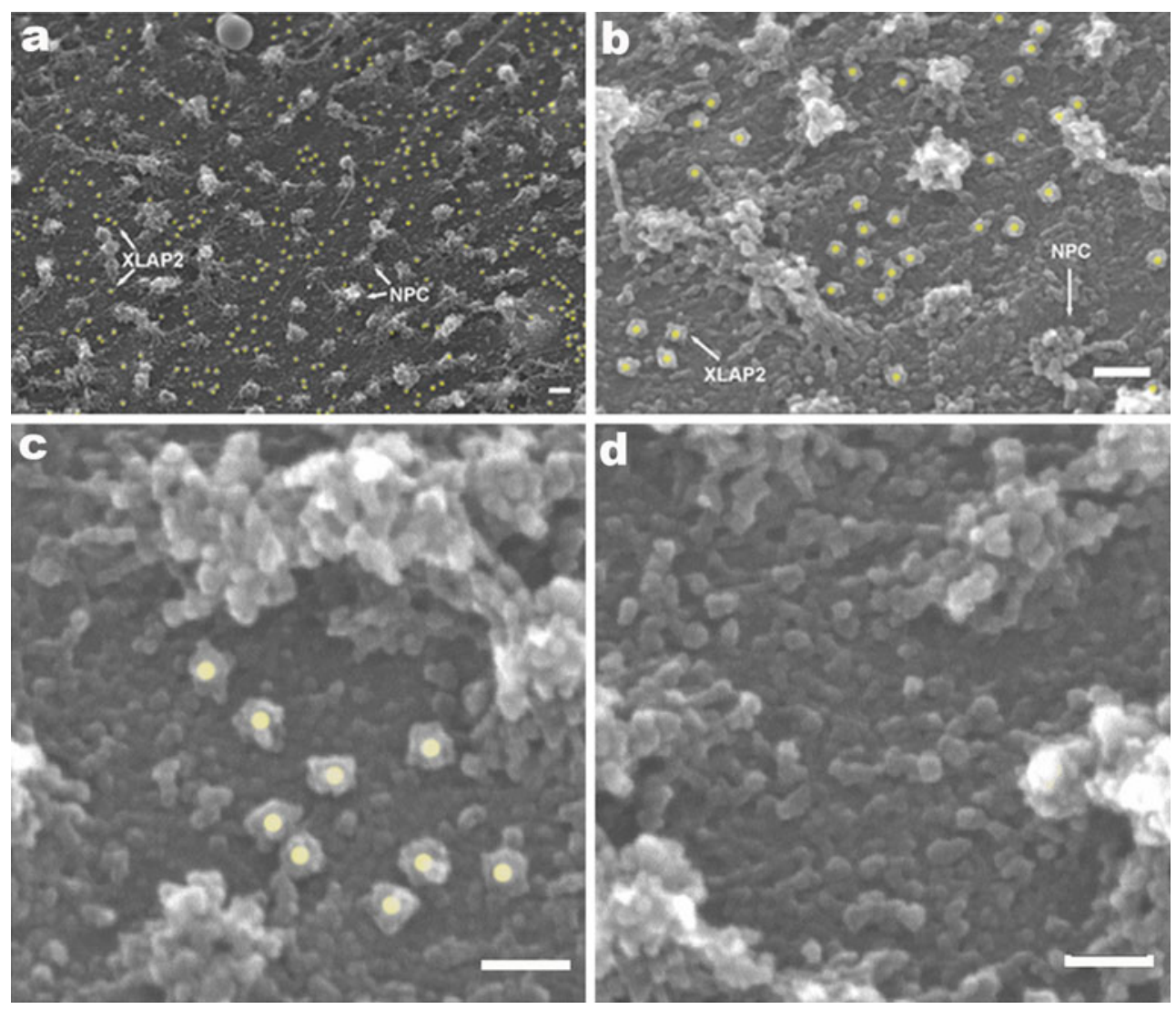

Fig. 5 Analyses of the distribution of XLAP2 $\omega$ and XLAP2 $\gamma$ proteins on the inner surface of the nuclear envelope (NE) of Xenopus oocyte by using immunogold labelling and scanning electron microscopy (SEM). Manually isolated nuclei from frog oocytes were transferred onto silicone chips. The inner side of the NE was manually exposed by gently removing the nuclear content and processing for SEM immunogold procedures. XLAP2 was detected with antibodies against XLAP2 followed by Protein A coupled with 10-nm gold

particles (yellow dots gold particles associated with immunocomplex bound to XLAP2 protein, NPC nuclear pore complexes). a, b Embryonic XLAP2 proteins localize non-randomly and show a tendency to form clusters and microdomains at the inner nuclear membrane. They also show a tendency to locate longitudinally; this might reflect the shape of the nuclear lamina filaments to which they are initially attached. c Typical cluster of XLAP2 proteins. d Typical region lacking XLAP2 protein. Bars $100 \mathrm{~nm}(\mathbf{a}, \mathbf{b}), 50 \mathrm{~nm}(\mathbf{c}, \mathbf{d})$ 
In adult tissues and in XTC cells, a fraction of XLAP2 $\beta$ has also been detected inside the nucleus, usually in clusters on heterochromatin, but also with no visible traces of $\mathrm{NE}$ invaginations being detected (Figs. 3, 4, Table 1). Immunogold labelling of XTC cells (Fig. 3f-j) suggests that this intra-nuclear fraction is much greater than the possible amount of proteolytic degradation products detected in XTC cells. This suggests the presence of a hydrophobic (lipid) fraction associated with the surface of chromatin as reported for the first time some time ago (Maraldi et al. 1992) or might reflect tubular NE invaginations (Ellenberg et al. 1997; Fricker et al. 1997; Gehrig et al. 2007) or both. The last-mentioned seems to be the most probable, since our confocal microscopy data indicate the presence of the XLAP2 $\beta$ fraction inside the cell nucleus. This is especially visible during prophase when the chromatin is condensing and relocating some chromatin components or adjacent proteins (Fig. 3c). Based on the TEM immunogold results (Table 1) and three-dimensional analyses of confocal sections through XTC cell nuclei (Fig. 4), this intranuclear fraction of XLAP2 can be divided into membranefraction-associated with NE invaginations (and chromatin) and non-membrane-fraction-associated with chromatin.

The calculated RLI data confirm that only some of the clusters of intra-nuclear XLAP2 $\beta$ are connected with NE invaginations (Table 1). The difference in labelling intensity between chromatin at the $\mathrm{NE}$ and at the invaginations might be the result of incorrect assumptions of the number of invaginations in single nuclei (three per nucleus, as in Fig. 3d). If we take into account only those invaginations with a visible lumen, make appropriate corrections for this in the calculations of RLI and assume one invagination per cell nucleus, we will obtain an RLI value for chromatin at the invaginations similar to the value for NE-associated XLAP2. This correlates with the same intensity of the signal from XLAP $2 \beta$-stained NE and from XLAP $2 \beta$ stained NE invaginations detected by confocal microscopy (Fig. 4b, f). The association of all isoforms of XLAP2 in clusters on chromatin and/or NE is of great interest. It confirms that XLAP2 proteins are involved in the organization of chromatin structure by anchoring large protein complexes and thereby modifying chromatin structure on a domain-like scale rather than a single transcription unit.

The second implication is the possible involvement of LAP2 protein clusters in forming discrete microdomains at the NE composed of either lamin A or lamin B. Newly imported into the nucleus, lamin A and B molecules are not incorporated uniformly into the nuclear lamina but form separate microdomains (Furukawa et al. 2009) reflecting separate microdomains of lamin $\mathrm{A}$ and $\mathrm{B}$ present in the nuclear lamina (Haraguchi et al. 2008). Our data indicate that these lamin A and lamin B microdomains are formed and stabilized by interactions with LEM domain proteins of the inner nuclear membrane, such as XLAP2. Since we have observed the full colocalization between XLAP2 isoforms and B-type lamins (Supplemental Fig. 2), we suggest that part of the lamin B microdomains in Xenopus NE might be formed and maintained by LAP 2 proteins (e.g. Fig. 4c, d). XLAP2 proteins have been found to coprecipitate with A- and B-type lamins in multiprotein complexes (Lang and Krohne 2003) and so the specificity of particular microdomain formed by XLAP2 might be determined by other proteins of the complex specific either for A- or B-type lamins. Our data also suggest that XLAP2 proteins are similarly involved in linking epigenetically marked chromatin as other integral membrane proteins of the NE inner membrane, e.g lamin-B-receptor protein (Makatsori et al. 2004).

Acknowledgement We are grateful to Prof. Reimer Stick for the kind gift of antibodies against Xenopus lamin B2 and B2/B3 and for useful discussion. We also acknowledge the helpful assistance of Mrs. Christine Richardson and Dr. Georgia Salpingidou (Integrative Cell Biology Laboratory, University of Durham) with transmission electron microscopy (TEM) and confocal microscopy, respectively. Special thanks are due to Pawel Sopel (Jagiellonian University, Cracow) for help with statistical analyses and to the Department of Histology and Embryology, Wrocław Medical University for the TEM analyses of frog brain and muscle.

Open Access This article is distributed under the terms of the Creative Commons Attribution Noncommercial License which permits any noncommercial use, distribution, and reproduction in any medium, provided the original author(s) and source are credited.

\section{References}

Allen TD, Rutherford SA, Murray S, Sanderson HS, Gardiner F, Kiseleva E, Goldberg MW, Drummond SP (2007) A protocol for isolating Xenopus oocyte nuclear envelope for visualization and characterization by scanning electron microscopy (SEM) or transmission electron microscopy (TEM). Nat Protoc 2:11661172

Alsheimer M, Fecher E, Benavente R (1998) Nuclear envelope remodelling during rat spermiogenesis: distribution and expression pattern of LAP2/thymopoietins. J Cell Sci 111:2227-2234

Berger JM, Gamblin SJ, Harrison SC, Wang JC (1996) Structure and mechanism of DNA topoisomerase II. Nature 379:225-232

Cai M, Huang Y, Ghirlando R, Wilson KL, Craigie R, Clore GM (2001) Solution structure of the constant region of nuclear envelope protein LAP2 reveals two LEM-domain structures: one binds BAF and the other binds DNA. EMBO J 20:43994407

Dorner D, Gotzmann J, Foisner R (2007) Nucleoplasmic lamins and their interaction partners, LAP2alpha, Rb, and BAF, in transcriptional regulation.FEBS J 274:1362-1373

Ellenberg J, Siggia ED, Moreira JE, Smith CL, Presley JF, Worman HJ, Lippincott-Schwartz J (1997) Nuclear membrane dynamics and reassembly in living cells: targeting of an inner nuclear membrane protein in interphase and mitosis. J Cell Biol 138:1193-1206

Fricker M, Hollinshead M, White N, Vaux D (1997) Interphase nuclei of many mammalian cell types contain deep, dynamic, tubular 
membrane-bound invaginations of the nuclear envelope. J Cell Biol 136:531-544

Furukawa K (1999) LAP2 binding protein 1 (L2BP1/BAF) is a candidate mediator of LAP2-chromatin interaction. J Cell Sci 112:2485-2492

Furukawa K, Ishida K, Tsunoyama TA, Toda S, Osoda S, Horigome T, Fisher PA, Sugiyama S (2009) A-type and B-type lamins initiate layer assembly at distinct areas of the nuclear envelope in living cells. Exp Cell Res 315:1181-1189

Gant TM, Harris CA, Wilson KL (1999) Roles of LAP2 proteins in nuclear assembly and DNA replication: truncated LAP2beta proteins alter lamina assembly, envelope formation, nuclear size, and DNA replication efficiency in Xenopus laevis extracts. J Cell Biol 144:1083-1096

Gehrig KM, Cornell RB, Ridgway ND (2007) Expansion of the nucleoplasmic reticulum requires the coordinated activity of lamins and CTP:phosphocholine cytidylyltransferase (CCT) \{alpha\}. Mol Biol Cell E07:02-0179

Haraguchi T, Kojidani T, Koujin T, Shimi T, Osakada H, Mori C, Yamamot A, Hiraoka Y (2008) Live cell imaging and electron microscopy reveal dynamic processes of BAF-directed nuclear envelope assembly. J Cell Sci 121:2540-2554

Harris CA, Andryuk PJ, Cline S, Chan HK, Natarajan A, Siekierka JJ, Goldstein G (1994) Three distinct human thymopoietins are derived from alternatively spliced mRNAs. Proc Natl Acad Sci USA 91:6283-6287

Lang C, Krohne G (2003) Lamina-associated polypeptide 2beta (LAP2beta) is contained in a protein complex together with Aand B-type lamins. Eur J Cell Biol 82:143-153

Lang C, Paulin-Levasseur M, Gajewski A, Alsheimer M, Benavente R, Krohne G (1999) Molecular characterization and developmentally regulated expression of Xenopus lamina-associated polypeptide 2 (XLAP2). J Cell Sci 112:749-759

Makatsori D, Kourmouli N, Polioudaki H, Shultz LD, Mclean K, Theodoropoulos PA, Singh PB, Georgatos SD (2004) The inner nuclear membrane protein lamin $\mathrm{B}$ receptor forms distinct microdomains and links epigenetically marked chromatin to the nuclear envelope. J Biol Chem 279:25567-25573

Maraldi NM, Zini N, Squarzoni S, Del Coco R, Sabatelli P, Manzoli FA (1992) Intranuclear localization of phospholipids by ultrastructural cytochemistry. J Histochem Cytochem 40:1383-1392

Markiewicz E, Dechat T, Foisner R, Quinlan RA, Hutchison CJ (2002)Lamin A/C binding protein LAP2alpha is required for nuclear anchorage of retinoblastoma protein.Mol Biol Cell 13:4401-4413

Martins S, Eikvar S, Furukawa K, Collas P (2003) HA95 and LAP2 beta mediate a novel chromatin-nuclear envelope interaction implicated in initiation of DNA replication. J Cell Biol 160:177-188

Mayhew T (2005) How to count your gold: a tutorial on TEM immunogold label quantification. Microsc Anal 19:9-12

Meier J, Campbell KHS, Ford CC, Stick R, Hutchison CJ (1991) The role of lamin LIII in nuclear assembly and DNA replication, in cell-free extracts of Xenopus eggs. J Cell Sci 98:271-279

Nili E, Cojocaru GS, Kalma Y, Ginsberg D, Copeland NG, Gilbert DJ, Jenkins NA, Berger R, Shaklai S, Amariglio N, Brok-Simoni F, et al (2001) Nuclear membrane protein LAP2beta mediates transcriptional repression alone and together with its binding partner GCL (germ-cell-less). J Cell Sci 114:3297-3307

Nieuwkoop PD, Faber J (1994) The stages of Xenopus embryonic development. Normal table of Xenopus laevis (Daudin). North Holland, Amsterdam

O'Brien LL, Wiese C (2006) TPX is required for postmitotic nuclear assembly in cell-free Xenopus laevis egg extracts. J Cell Biol 173:685-694

Pekovic V, Harborth J, Broers JL, Ramaekers FC, Engelen B van, Lammens M, Zglinicki $\mathrm{T}$ von, Foisner R, Hutchison $\mathrm{C}$, Markiewicz E (2007)Nucleoplasmic LAP2alpha-lamin A complexes are required to maintain a proliferative state in human fibroblasts. J Cell Biol 176:163-172

Ralle T, Grund C, Franke WW, Stick R (2004) Intranuclear membrane structure formations by CaaX-containing nuclear proteins. J Cell Sci 117:6095-6104

Rzepecki R, Fisher PA (2000) During both interphase and mitosis, DNA topoisomerase II interacts with DNA as well as RNA through the protein's C-terminal domain. J Cell Sci 113:16351647

Rzepecki R, Bogachev SS, Kokoza E, Stuurman N, Fisher PA (1998) In vivo association of lamins with nucleic acids in Drosophila melanogaster. J Cell Sci 111:121-129

Salpingidou G, Rzepecki R, Kiseleva E, Lyon C, Lane B, Fusiek K, Golebiewska A, Drummond S, Allen T, Ellis JA, Smythe C, et al (2008) NEP-A and NEP-B both contribute to nuclear pore formation in Xenopus eggs and oocytes. J Cell Sci 121:706-716

Schirmer EC, Foisner R (2007)The lamina-associated polypeptide 2 (LAP2) isoforms beta, gamma and omega of zebrafish: developmental expression and behavior during the cell cycle. Exp Cell Res 313:2167-2179

Schoft VK, Beauvais AJ, Lang C, Gajewski A, Prufert K, Winkler C, Akimenko M-A, Paulin-Levasseur M, Krohne G (2003) The lamina-associated polypeptide 2 (LAP2) isoforms beta, gamma and omega of zebrafish: developmental expression and behavior during the cell cycle. J Cell Sci 116:2505-2517

Shaklai S, Somech R, Gal-Yam EN, Deshet-Unger N, MoshitchMoshkovitz S, Hirschberg K, Amariglio N, Simon AJ, Rechavi G (2008) LAP2zeta binds BAF and suppresses LAP2beta-mediated transcriptional repression.Eur J Cell Biol 87:267-278

Shumaker DK, Lee KK, Tanhehco YC, Craigie R, Wilson KL (2001) LAP2 binds to BAF-DNA complexes: requirement for the LEM domain and modulation by variable regions. EMBO J 20:1754-1764

Somech R, Shaklai S, Geller O, Amariglio N, Simon AJ, Rechavi G, Gal-Yam EN (2005) The nuclear-envelope protein and transcriptional repressor LAP2beta interacts with HDAC3 at the nuclear periphery, and induces histone $\mathrm{H} 4$ deacetylation. J Cell Sci 118:4017-4025

Wagner N, Krohne G (2007) LEM-domain proteins: new insights into lamin-interacting proteins.Int Rev Cytol 261:1-46

Yang L, Guan T, Gerace L (1997) Lamin-binding fragment of LAP2 inhibits increase in nuclear volume during the cell cycle and progression into S phase.J Cell Biol 139:1077-1087

Zaremba-Czogalla M, Dubinska-Magiera M, Rzepecki R (2011) Laminopathies - the molecular background of disease and prospects for treatment. Cell Mol Biol Lett 16:114-148 